\title{
Crystal Structure Prediction of Magnetic Transition-Metal Oxides by Using Evolutionary Algorithm and Hybrid DFT Methods
}

\author{
Mikhail S. Kuklin*() and Antti J. Karttunen ${ }^{(1)}$ \\ Department of Chemistry and Materials Science, Aalto University, P.O. Box 16100, FI-00076 Aalto, Finland \\ Supporting Information
}

ABSTRACT: Although numerous crystal structures have been successfully predicted by using currently available computational techniques, prediction of strongly correlated systems such as transition-metal oxides remains a challenge. To overcome this problem, we have interfaced evolutionary algorithm-based USPEX method with the CRYSTAL code, enabling the use of Gaussian-type localized atomic basis sets and hybrid density functional (DFT) methods for the prediction of crystal structures. We report successful crystal structure predictions of several transition-metal oxides $(\mathrm{NiO}$, $\mathrm{CoO}, \alpha-\mathrm{Fe}_{2} \mathrm{O}_{3}, \mathrm{~V}_{2} \mathrm{O}_{3}$, and $\mathrm{CuO}$ ) with correct atomic magnetic moments, spin configurations, and structures by using the USPEX method in combination with the CRYSTAL code and Perdew-Burke-Ernzerhof (PBE0) hybrid functional. Our benchmarking results demonstrate that USPEX + hybrid DFT is a suitable combination to reliably predict the magnetic structures of strongly correlated materials.

\section{INTRODUCTION}

To tune the physical properties of any material with high precision, one has to understand the atomic-level structure of the material. When there are no crystals suitable for singlecrystal structure determination, the crystal structure can often still be solved from an X-ray powder-diffraction pattern. However, if the experimental data quality is poor or the structure has a completely new structure type, the complete structure solution from the powder data becomes very difficult. For magnetic materials, the magnetic spin structure further complicates the full structure solution and neutron diffraction has to be used to obtain the information of spin configurations. To speed up structure solution of new materials and materials design process in general, different computational crystal structure prediction algorithms have been developed. ${ }^{1-5}$ Even though some major successes have already been achieved, crystal structure prediction has been regarded for a long time as one of the most challenging problems in material sciences. ${ }^{6,7}$ In particular, the prediction of magnetic ground states remains essentially an unsolved problem. This is due to the complicated nature of the magnetic compounds, where spin configurations and the magnetic unit cell have to be taken into account in the structure predictions.

One important class of magnetic crystal structures is the transition-metal oxides. Transition-metal oxides play a crucial role in a wide range of applications from catalysis to electronics. $^{8-14}$ It is well known that standard generalized gradient approximation (GGA) functionals such as PerdewBurke-Ernzerhof (PBE) fail to correctly treat the magnetic moments and electronic structure of systems such as strongly

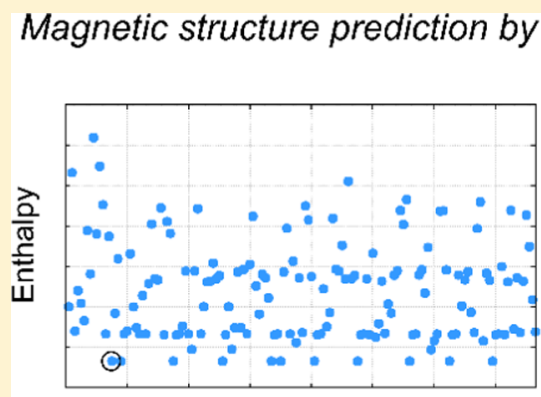

Structure number

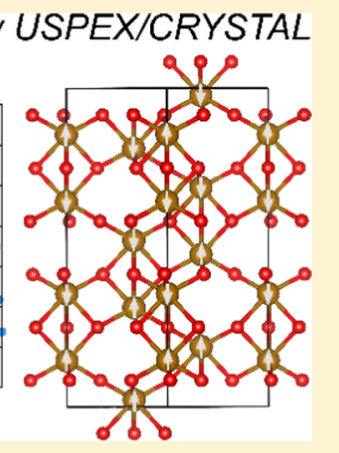

correlated transition-metal oxides, sometimes even favoring a wrong magnetic ground state. ${ }^{15-22}$ Even in the case of nonmagnetic transition-metal oxides such as $\mathrm{Cu}_{2} \mathrm{O}$, the band structure may not be correctly described by GGA functionals. $^{23}$ The reason is the self-interaction error of GGA, which results in the over-delocalization of the electrons on transition-metal $3 \mathrm{~d}$ and oxygen $2 \mathrm{p}$ orbitals. ${ }^{15,24-27}$ Consequently, this results in an electronic structure that can be even qualitatively wrong. The problem with the delocalization of $3 \mathrm{~d}$ orbitals can be overcome by using the Hubbard $U$ parameter to localize the electrons on the transition-metal atoms, but this does not solve the problem related to the treatment of oxygen $2 p$ orbitals. In particular, recent work clearly demonstrates that hybrid functionals exclusively localize density onto the $2 \mathrm{p}$ orbitals, whereas hybrid density functional (DFT) methods $+U$ does not necessarily do the same. ${ }^{28}$ Therefore, a wrongly chosen $U$ value may not provide any improvements over standard DFT-GGA functionals favoring a wrong magnetic state. Furthermore, even GGA $+U$ still underestimates band gaps of transition-metal monoxides. ${ }^{24,29}$ Also, when the goal is to predict new crystal structures, it is not clear what particular $U$ value should be used if the material is entirely new. ${ }^{25,29}$ The problems related to the $U$ correction can be solved by adding exact exchange and using hybrid DFT which equally improves localization on transition metal and oxygen atoms and has an impact on the relative position of the

Received: August 24, 2018

Revised: October 10, 2018

Published: October 11, 2018 
energy levels of transition metal d and oxygen p states. ${ }^{21,24,26,27}$ One of the major differences between the hybrid functionals and GGA $+U$ scheme is that the amount of the exact exchange is not tuned for each material in the case of hybrid DFT. Furthermore, it has been proven that the hybrid functionals with $\sim 20 \%$ of the exact exchange reliably describe magnetic properties of transition-metal oxides. ${ }^{21,24,27,28}$

From a technical point of view, an algorithm for crystal structure prediction has to satisfy the following criteria: (i) computationally not too expensive, (ii) automation of all stages, and (iii) usable for different kinds of systems. The examples of some methods developed to predict crystal structures are simulated annealing, ${ }^{1}$ minima hopping, ${ }^{2,4}$ and metadynamics. ${ }^{3,5}$ Although many successes have been achieved, there are still many known cases where structures have not been predicted correctly. ${ }^{6}$ This is mainly due to the following problems: (1) the need to have a good starting geometry from which the algorithm starts the search, (2) complicated choice of the initial input parameters, (3) slow performance, and (4) repeating visits to already investigated minima.

Methods based on evolutionary algorithms (EA) represent one of the most successful approaches to search for global minima and to predict unknown crystal structures. ${ }^{30-34}$ EA methods have the following advantages: (1) they do not require any experimental parameterization; (2) they are selfdeveloping, as the evolutionary algorithm forces the population to improve from generation to generation; (3) they provide accurate results as long as ab initio methods, usually DFT, are used for local optimization of each candidate structure; and (4) they learn from history, thus avoiding recalculation of already studied structures. In particular, USPEX (Universal Structure Predictor: Evolutionary Xtallography) is an EA code that has been successfully used in many different applications. ${ }^{32-39}$ The First Blind Test for inorganic crystal structure predictions demonstrated that USPEX is a robust and advanced method. ${ }^{32,35,39,40}$ USPEX includes many advanced features: (1) cell reduction techniques and (2) constraints on bond lengths and angles that prevent construction of flat cells and majority of unreasonable structures; (3) cell splitting techniques that prevent energetically poor structures in the initial population; (4) local ordering that represents a smart way to construct new candidates from previously studied structures; (5) fingerprint function that avoids trapping in local minima.

USPEX is already interfaced with many DFT codes, but these are typically based on plane-wave basis sets and therefore known to have relatively high computational cost for hybrid DFT methods. CRYSTAL program package that utilizes local atomic basis sets is known to be an effective code for employing hybrid functionals. $^{41}$ Therefore, we decided to develop a CRYSTAL interface for USPEX to produce a suitable DFT-based EA approach for the prediction of magnetic crystal structures.

In this paper, we describe the first successful crystal structure prediction benchmarking results for magnetic binary transitionmetal oxides. We apply a new CRYSTAL interface for USPEX code to investigate $\mathrm{NiO}, \mathrm{CoO}, \alpha-\mathrm{Fe}_{2} \mathrm{O}_{3}, \mathrm{~V}_{2} \mathrm{O}_{3}$, and $\mathrm{CuO}$. To show that this approach works equally well for nonmagnetic structures, we investigate the crystal structure of $\mathrm{Cu}_{2} \mathrm{O}$.

\section{COMPUTATIONAL DETAILS}

2.1. Electronic Structure Calculations. Crystal structure predictions were carried out by using USPEX 9.4.4 code. ${ }^{33-35}$ All quantum chemical calculations within the USPEX simulations were performed using the CRYSTAL17 code. $^{41}$ We developed a new CRYSTAL interface for USPEX that allows using CRYSTAL for the local optimization of candidate structures. The interface is written in MATLAB and integrated with a development version of USPEX. The interface will be included in a forthcoming release of USPEX. Hybrid PBE0DFT functional with 25\% Hartree-Fock and 75\% PBE exchange was utilized in the study. ${ }^{42,43}$ All-electron, Gaussian-type split-valence + polarization (SVP) level basis sets based on Karlsruhe def2-SVP basis sets were used within the crystal structure predictions (a list of all used basis sets is given in the Supporting Information). ${ }^{44}$ The lowest-energy structures from each USPEX simulation were reoptimized by using triple- $\zeta$-valence + polarization (TZVP) level basis sets. The Karlsruhe basis sets are known to be among the best molecular basis sets, and by introducing only minor modifications into them to make periodic calculations feasible, we can approach the choice of basis set in a systematic way. $\mathrm{CoO}$ structures could only be reoptimized with the SVP basis set due SCF convergence problems in the case of a TZVP basis set. For the final optimization after the USPEX run, the used $k$ point meshes were $8 \times 8 \times 8$ for all studied structures. Spinunrestricted formalism was used for all calculations of magnetic structures. Full computational details of CRYSTAL calculations can be found in the Supporting Information. For the final optimization after the USPEX run, tightened tolerance factors (TOLINTEG) of $8,8,8,8$, and 16 were used for the evaluation of the Coulomb and exchange integrals. This means that if the overlap between two atomic orbitals is smaller than $10^{-8}$ (or $10^{-16}$ for some integrals), the corresponding integral is disregarded (see ref 45 for detailed explanation of the TOLINTEG criteria). Because the initial population is based on random structure generation, each USPEX run will be different. Therefore, USPEX structure searches were carried out twice for each studied structure to confirm the robustness of the present CRYSTAL + USPEX methodology (USPEX input files are given in the Supporting Information). One has to keep in mind that hybrid DFT is computationally more expensive than GGA or GGA + U. In general, the computational cost is determined by the cost of the local optimizations. Therefore, it is better to use GGA functionals for systems such as metals which are well-described with DFTGGA.

2.2. Working Principles of the USPEX. A typical USPEX workflow is illustrated in Figure 1.

USPEX first randomly generates a set of structures known as candidate structures. These structures are called a generation or, depending on the context, population. We use the term generation when discussing a particular set of the structures, for example, from generation 1 . The term population refers to all considered candidate structures within one single USPEX structure prediction run. After random generation, the candidate structures are locally optimized by an ab initio code (here, CRYSTAL). When the local optimizations of all structures in the generation are finished, the next generation has to be built by using variation operators on the lowestenergy structures from the previous generation. Furthermore, a small number of randomly generated structures are added to 


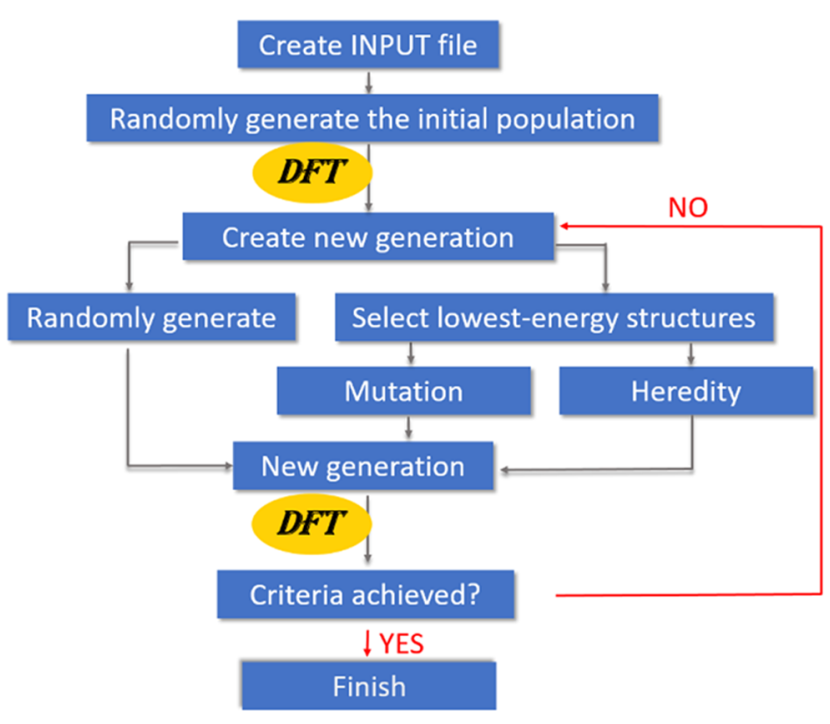

Figure 1. Typical USPEX workflow for crystal structure prediction.

diversify the population. USPEX uses the following variation operators: heredity, permutation, lattice mutation, and atomic mutations (softmutation and coormutation). Next, the structures from the new generation are locally optimized and the above-described process is repeated until halting criterion is met. Usually, USPEX stops when the same lowest-energy structure is produced in several generations in a row. Overall, a minimal USPEX input file contains (1) atom types and the amount of each element, (2) size and number of generations, (3) parameters of the USPEX algorithm, for example, the variation operators used (present USPEX input files are given in the Supporting Information).

\section{RESULTS AND DISCUSSION}

The results will be discussed as follows. We start with resulcts on the nonmagnetic $\mathrm{Cu}_{2} \mathrm{O}$ structure by giving the description of the prediction procedure by USPEX evolutionary algorithm and comparing the calculated structure with experimental data. Next, we discuss magnetic transition-metal oxides in the same way as $\mathrm{Cu}_{2} \mathrm{O}$ in the following order: $\mathrm{NiO}, \mathrm{CoO}, \alpha-\mathrm{Fe}_{2} \mathrm{O}_{3}$, $\mathrm{V}_{2} \mathrm{O}_{3}$, and $\mathrm{CuO}$.

3.1. $\mathrm{Cu}_{2} \mathrm{O}$. Copper(I) oxide $\mathrm{Cu}_{2} \mathrm{O}$ has a cubic crystal structure with $P n \overline{3} m$ space group $(224){ }^{46,47} \mathrm{Cu}_{2} \mathrm{O}$ is a nonmagnetic structure with the unit cell containing two formula units $\left(\mathrm{Cu}_{4} \mathrm{O}_{2}\right)$ and linearly coordinated copper atoms with oxygen atoms, which are, in turn, tetrahedrally coordinated (Figure 2).

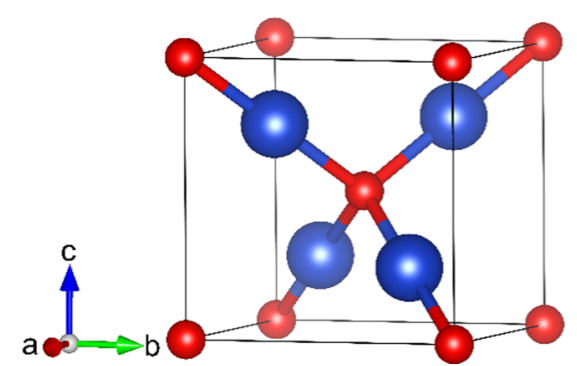

Figure 2. Lowest-energy unit cell predicted for $\mathrm{Cu}_{2} \mathrm{O}$ by USPEX (red: oxygen, blue: copper).
$\mathrm{Cu}_{4} \mathrm{O}_{2}$ composition was specified in the USPEX, corresponding to the $\mathrm{Cu}_{2} \mathrm{O}$ unit cell. Figure 3 illustrates the enthalpy per atom of all candidate structures as a function of structure number in two USPEX runs.

Hundred and forty-five structures were considered within 10 generations in the first USPEX simulation (Figure 3a). The second USPEX simulation screened 151 candidates within 10 generations (Figure $3 \mathrm{~b}$ ). However, the correct $\mathrm{Cu}_{2} \mathrm{O}$ crystal structure was found already in generation 4 in the first USPEX simulation and in generation 3 in the second run. As the local optimizations within USPEX are carried by using relatively weak convergence criteria and small basis sets to accelerate the evolutionary run, one typically has to reoptimize lowest-energy structures with a higher level of theory afterward. The results for the reoptimized lowest-energy structures are depicted in Table 1.

It is seen that structural properties of the predicted $\mathrm{Cu}_{2} \mathrm{O}$ structure are in line with the experimental data. The calculated lattice constant is only $1.2 \%$ larger than the experimental value. $\mathrm{Cu}-\mathrm{O}$ distance in the calculated structure also correlates very well with the experimental value $(1.87 \AA$ calc. and $1.85 \AA$ exp.). ${ }^{46,47}$ The calculated band gap $(2.3 \mathrm{eV})$ of $\mathrm{Cu}_{2} \mathrm{O}$ was found to be very close to experimental one of $2.2 \mathrm{eV}$. Next, we moved to structure predictions of magnetic transition-metal oxides.

3.2. NiO. Nickel(II) oxide (NiO) crystallizes in the cubic $F m \overline{3} m$ space group. The $\mathrm{Ni}^{2+}$ ions in the structure have an octahedral environment $(\mathrm{NaCl}$ structure type). The ground state of $\mathrm{NiO}$ is known to be antiferromagnetic (AFM) below the Néel temperature of $525 \mathrm{~K}$. The antiferromagnetic ground state can be realized in the subgroup $R \overline{3} m$ (166), where the nickel atoms with the opposite spin are located in adjacent layers along the [111] direction of the original face-centered cubic cell (Figure 4)..$^{50,51,56,70}$ The antiferromagnetic $\mathrm{NiO}$ structure shown in Figure 4 was also been found to be the most stable one by using different DFT functionals. ${ }^{21}$

In the case of a completely new material with unknown magnetic structure, we would have to check all possible magnetic states of the structures when comparing their stabilities. Here, the magnetic ground states are known from experiment. Therefore, we did not investigate all possible spin configurations but focused instead on the correct prediction of the spin configuration and the space group. This is made possible by use of hybrid functionals, as they reliably describe not only the geometry but also the magnetic properties of the transition-metal oxides. In the case of $\mathrm{NiO}$, it is known that hybrid DFT methods correctly give the AFM spin configuration shown in Figure 4 as the lowest-energy NiO structure; therefore, we did not carry out structure predictions for other spin configurations such as ferromagnetic ordering. ${ }^{21}$ In a general case, one should run the evolutionary search for a number of spin settings to find the ground-state spin configuration.

To predict an antiferromagnetic structure for $\mathrm{NiO}$, at least two formula units $\left(\mathrm{Ni}_{2} \mathrm{O}_{2}\right)$ must be used in the USPEX search. As for $\mathrm{Cu}_{2} \mathrm{O}$, two USPEX simulations were carried out (Figure 5).

In general, 124 and 123 candidate structures within 10 generations were considered in the first (Figure 5a) and the second (Figure 5b) USPEX simulations, respectively. The correct $\mathrm{NiO}$ structure was identified in the first generation for both simulations. On the basis of Table 1, we see that the properties of the predicted structures are consistent with 

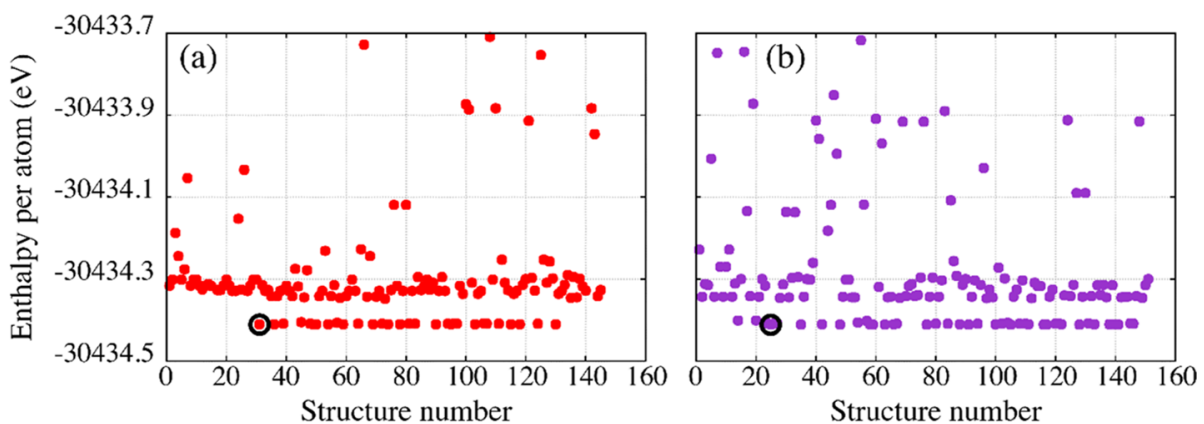

Figure 3. USPEX evolutionary crystal structure prediction of $\mathrm{Cu}_{2} \mathrm{O}$ (six atoms in the unit cell), showing the enthalpy per atom of all candidate structures along the evolutionary trajectory. Circle shows the first occurrence of the final global minimum. Plot (a) is the result of the first USPEX simulation, and plot (b) is the result of the second USPEX simulation.

Table 1. Magnetic Moments $\left(\mu_{\mathrm{B}} /\right.$ Transition-Metal Atom), Lattice Parameters $(\AA)$, Bond Lengths $(\AA)$, and Band Gaps (eV) of the Predicted Crystal Structures in Comparison with Experimental Data

\begin{tabular}{|c|c|c|c|c|c|c|c|c|c|c|}
\hline \multirow[b]{3}{*}{ oxide } & \multirow{2}{*}{\multicolumn{2}{|c|}{$\mu_{\mathrm{B}}$}} & \multicolumn{6}{|c|}{ lattice parameters $(\AA)$} & \multirow{2}{*}{\multicolumn{2}{|c|}{ band gap $(\mathrm{eV})$}} \\
\hline & & & & $a$ & & & & $c$ & & \\
\hline & calc. & exp. & calc. & exp. & calc. & exp. & calc. & exp. & calc. & exp. \\
\hline $\mathrm{Cu}_{2} \mathrm{O}$ & & & 4.318 & $4.269^{46,47}$ & & & & & 2.3 & $2.2^{47}$ \\
\hline $\mathrm{NiO}$ & 1.67 & $1.64,^{48} 1.77,^{49} 1.90^{50}$ & 4.187 & $4.177^{51}$ & & & & & $5.2^{b}$ & $4.0,{ }^{52} 4.3^{53}$ \\
\hline $\mathrm{CoO}^{a}$ & 2.74 & $3.35,3.8^{54,55}$ & 4.247 & $4.263^{56}$ & & & & & $4.7^{b}$ & $2.6^{57}$ \\
\hline$\alpha-\mathrm{Fe}_{2} \mathrm{O}_{3}$ & 4.24 & $4.6-5.2^{58}$ & 5.054 & $5.035^{59,60}$ & & & 13.728 & $13.747^{59,60}$ & $4.0^{b}$ & $2.16^{61,62}$ \\
\hline $\mathrm{V}_{2} \mathrm{O}_{3}$ & 2.02 & & 5.053 & $4.949^{63}$ & & & 13.824 & $13.998^{63}$ & 3.0 & \\
\hline $\mathrm{CuO}$ & 0.63 & $0.65,^{64} 0.68^{65,66}$ & 4.731 & $4.684^{67}$ & 3.436 & $3.423^{67}$ & 5.147 & $5.129^{67}$ & $3.4^{b}$ & $1.7^{68}$ \\
\hline
\end{tabular}

${ }^{a}$ An orbital moment, which is about $1 \mu_{\mathrm{B}}$, is not taken into account in the calculated result. ${ }^{69}{ }^{b}$ Band gaps for the $\alpha$ and $\beta$ spin are the same.
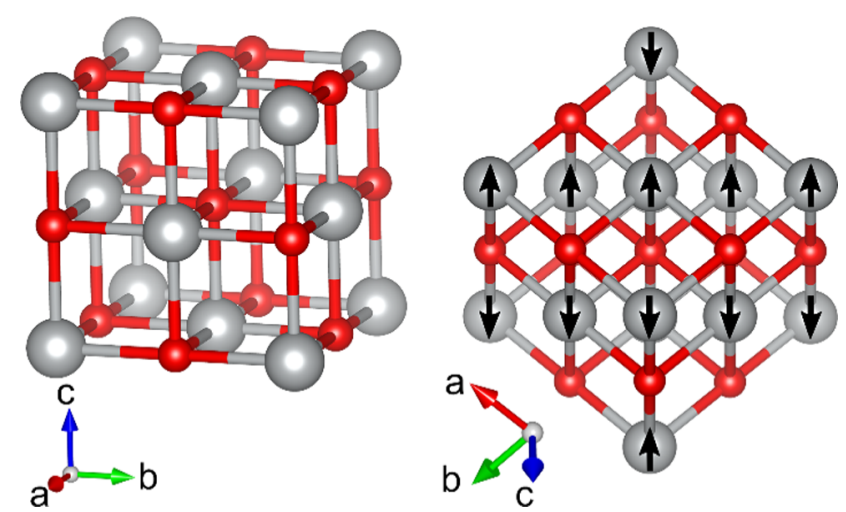

Figure 4. Lowest-energy unit cell predicted for $\mathrm{NiO}$ by USPEX (red: oxygen, gray: nickel). The directions of the magnetic moments are illustrated by arrows. experimental findings. The difference between the calculated and experimental lattice constant is very small $(+0.2 \%) .^{51}$ Calculated magnetic moment $\left(1.67 \mu_{\mathrm{B}}\right)$ reproduces the experimental value. ${ }^{48-50}$ All reported magnetic moments in the paper are spin-only values, meaning that orbital magnetic moments can result in some differences between experimental and computational results. The band gap of $\mathrm{NiO}$ is overestimated by about $1 \mathrm{eV}$. The main improvement of the hybrid functionals over GGA functionals is the correct treatment of valence bands and the states near the Fermi level, which leads to localization of atomic orbitals. Therefore, even though the band gaps of some structures are not reproduced quantitatively, the hybrid functional has a crucial impact on the quality of the results. In particular, it has been shown that the electronic properties and the geometry of $\mathrm{NiO}$ are reliably described with hybrid DFT methods even though the band gap is overestimated. ${ }^{14,71}$ For comparison, GGA-PBE
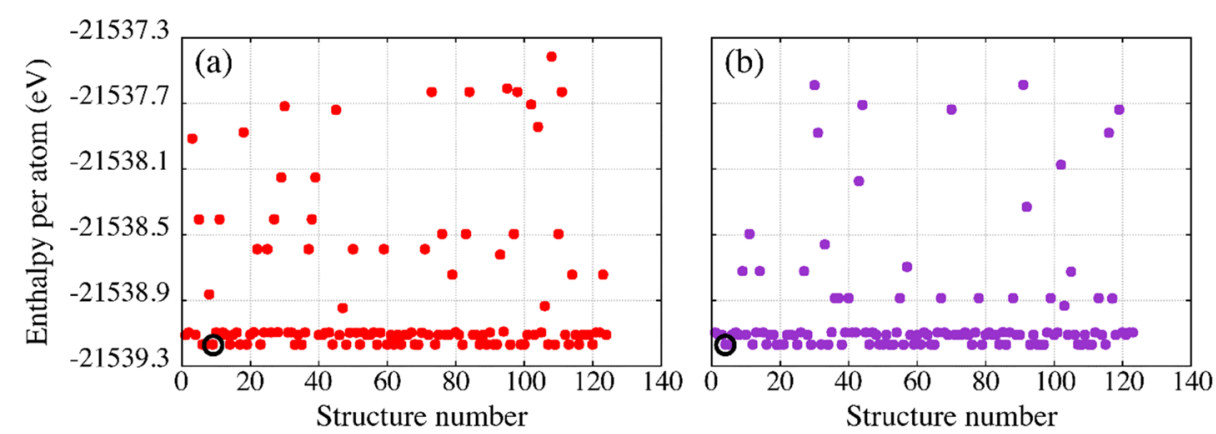

Figure 5. USPEX evolutionary crystal structure prediction of $\mathrm{NiO}$ (four atoms in the unit cell), showing enthalpy per atom of all candidate structures along the evolutionary trajectory. Circle shows the first occurrence of the final global minimum. Plot (a) is the result of the first USPEX simulation and plot (b) is the result of the second USPEX simulation. 

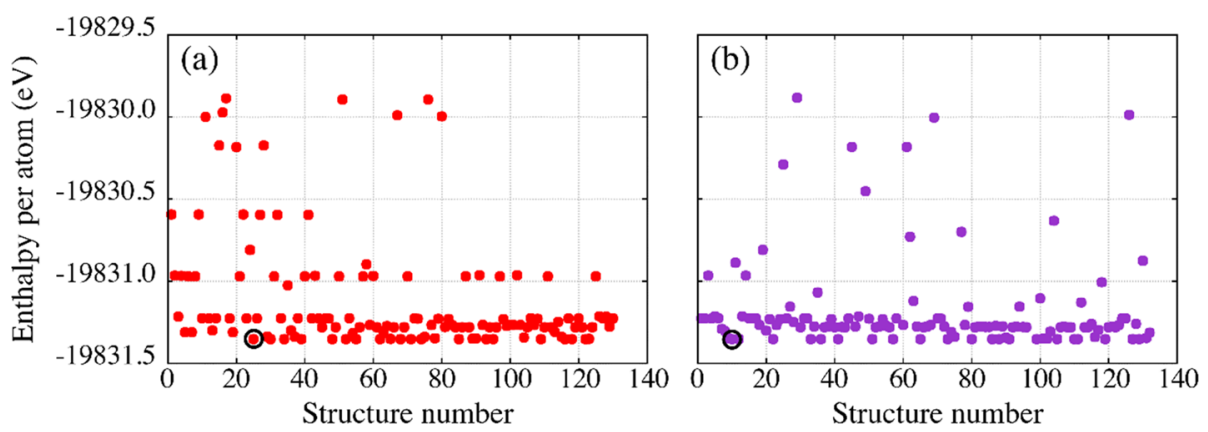

Figure 6. USPEX evolutionary prediction of the crystal structure of $\mathrm{CoO}$ (four atoms in the unit cell), showing enthalpy per atom of all candidate structures along the evolutionary trajectory. Circle shows the first occurrence of the final global minimum. Plot (a) is the result of the first USPEX simulation, and plot (b) is the result of the second USPEX simulation.

functional severely underestimates the band gap of $\mathrm{NiO}$, predicting a value of only $0.5 \mathrm{eV} .^{21}$ An interesting possibility for further improvement is the utilization of self-consistent hybrids, as implemented in CRYSTAL, in which case the amount of exact exchange is self-consistently obtained for different types of materials. ${ }^{72,73}$ However, at the moment, these approaches are computationally somewhat too expensive to be combined with evolutionary algorithm predictions.

3.3. CoO. Cobalt(II) oxide ( $\mathrm{CoO})$ adopts the same rocksalt structure as that of $\mathrm{NiO}$ (Figure 4), and it has AFM spinordering at its ground state below the Neel temperature of 293 $\mathrm{K}^{54-56}$ The same AFM configuration was found to be the most stable in previous DFT studies. ${ }^{21}$ The magnetic primitive unit cell contains two formula units $\left(\mathrm{Co}_{2} \mathrm{O}_{2}\right)$. The results of the two USPEX simulations are given in Figure 6.

Overall, 130 and 132 candidate structures were screened in the first (Figure 6a) and the second (Figure 6b) USPEX runs, respectively. This required 12 and 10 generations, respectively. The correct $\mathrm{CoO}$ structure was found in the generation 3 in the first USPEX simulation and in the generation 1 in the second run. As seen in Table 1, all properties are also in line with experimental findings. The difference in magnetic moment is due to the orbital moment: the experimental value is always a combination of spin and orbital moments, whereas our calculations do not take orbital moment into account. However, orbital moment has been estimated to be 1 $\mu_{\mathrm{B}}$ using local spin density approximation $+U$. Together with this orbital moment our calculated spin moment reproduces the experimental value. ${ }^{69}$ The optimized lattice parameter is almost identical to the experimental value with a difference only of $-0.4 \%{ }^{56}$ Band gap is overestimated by $2.1 \mathrm{eV}$. After these high-symmetry tests on $\mathrm{NiO}$ and $\mathrm{CoO}$, we moved to more complicated cases: $\alpha-\mathrm{Fe}_{2} \mathrm{O}_{3}$ (hematite) and $\mathrm{V}_{2} \mathrm{O}_{3}$ (corundum structure).

3.4. $\alpha-\mathrm{Fe}_{2} \mathrm{O}_{3}$. Iron(III) oxide (hematite) has trigonal corundum structure with $R \overline{3} c$ space group (167) when magnetic ordering is not taken into account. ${ }^{59-61,74}$ The magnetic unit cell contains two formula units $\left(\mathrm{Fe}_{4} \mathrm{O}_{6}\right)$, and it is known to be stable in an AFM configuration below the Néel temperature of $955 \mathrm{~K}^{22,74}$ For the unit cell with the AFM spinordering, the symmetry is reduced to the subgroup $R \overline{3}$ (148). Each Fe atom is octahedrally coordinated with six $\mathrm{O}$ atoms that form close-packed planes (Figure 7). The AFM hematite structure has been also found to be the lowest-energy structure by DFT methods. ${ }^{22}$

Hundred and fifty-three structures within 10 generations were screened in the first USPEX run (Figure 8a), and 185

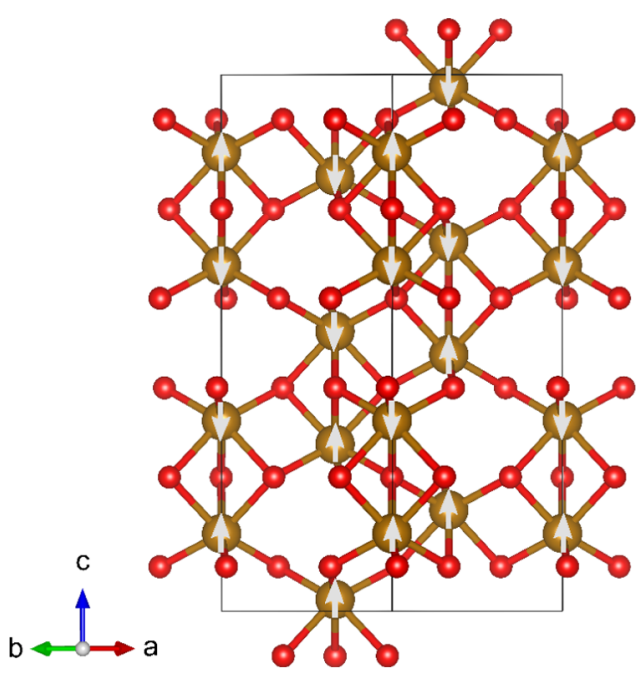

Figure 7. Lowest-energy unit cell for $\alpha$ - $\mathrm{Fe}_{2} \mathrm{O}_{3}$ predicted by USPEX (red: oxygen, brown: iron). The directions of the magnetic moments are illustrated by arrows.

structures in 10 generations were calculated during the second simulation (Figure $8 b$ ). The hematite structure in the AFM state was found in generations 1 and 4 for the first and second USPEX simulations, respectively.

The calculated magnetic moments of the Fe atoms (Table 1) are very close to the experimental values. The geometrical properties of the predicted hematite crystal structure match experimental data very well (Table 1 ): lattice constants are different by +0.4 and $-0.2 \%$ for $a$ and $c$, respectively. The $c / a$ ratio is also in line with experimental findings: 2.716 calc. and 2.730 exp. $^{59,60} \mathrm{Fe}-\mathrm{Fe}$ distances in the calculated structure are found to be 2.92 and $3.94 \AA$, whereas the experimental values are 2.88 and $3.98 \AA$. Band gap is overestimated by $1.8 \mathrm{eV}$. Importantly, hematite can have different spin configurations in the AFM state: $(++--),(+-+-)$, and $(+--+)$, where + and - designate $\mathrm{Fe}$ spin up and spin down along the $c$-axis for the leftmost atoms in Figure 7 . The most stable hematite structure predicted by USPEX corresponds to $(+--+)$ magnetic configuration, which is line with previous studies. ${ }^{22,74}$ For curiosity, we additionally calculated $(+-+-)$ hematite configuration at the PBE0/TZVP level of theory and it was found to be less stable than $(+--+)$ configuration by $5.6 \mathrm{~kJ} /$ $\mathrm{mol}$ per atom $(56 \mathrm{~kJ} / \mathrm{mol}$ per unit cell).

3.5. $\mathbf{V}_{2} \mathbf{O}_{3}$. Vanadium(III) oxide crystallizes in the trigonal corundum structure with $\bar{R} \overline{3} c$ space group (167) in the case of nonmagnetic unit cell and $R 3 c$ (161) for the magnetically 

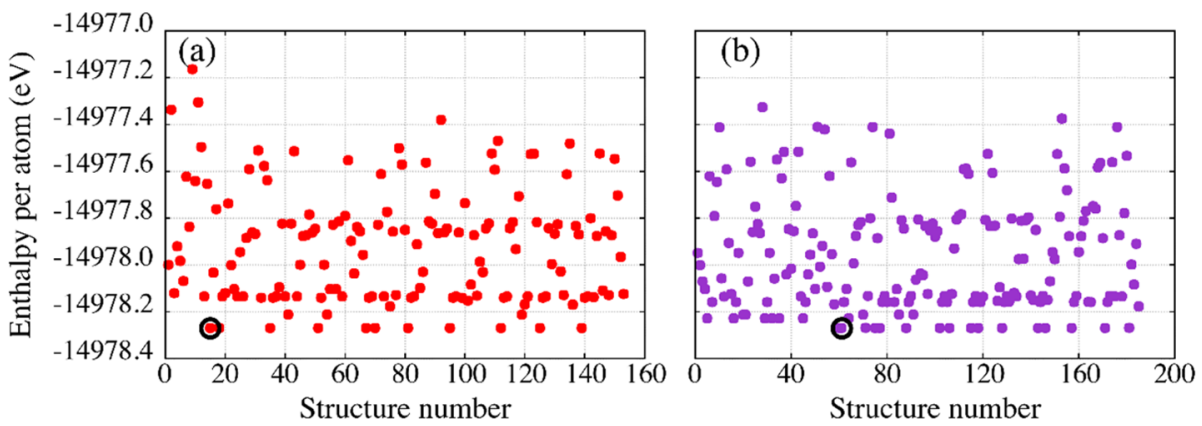

Figure 8. USPEX evolutionary crystal structure prediction of $\alpha$ - $\mathrm{Fe}_{2} \mathrm{O}_{3}$ (10 atoms in the unit cell), showing enthalpy per atom of all candidate structures along the evolutionary trajectory. The circle shows the first occurrence of the final global minimum. Plot (a) is the result of the first USPEX simulation, and plot (b) is the result of the second USPEX simulation.

ordered structure (Figure 9). ${ }^{60,75,76}$ The unit cell contains two formula units $\left(\mathrm{V}_{4} \mathrm{O}_{6}\right)$. In fact, it is known from experiments

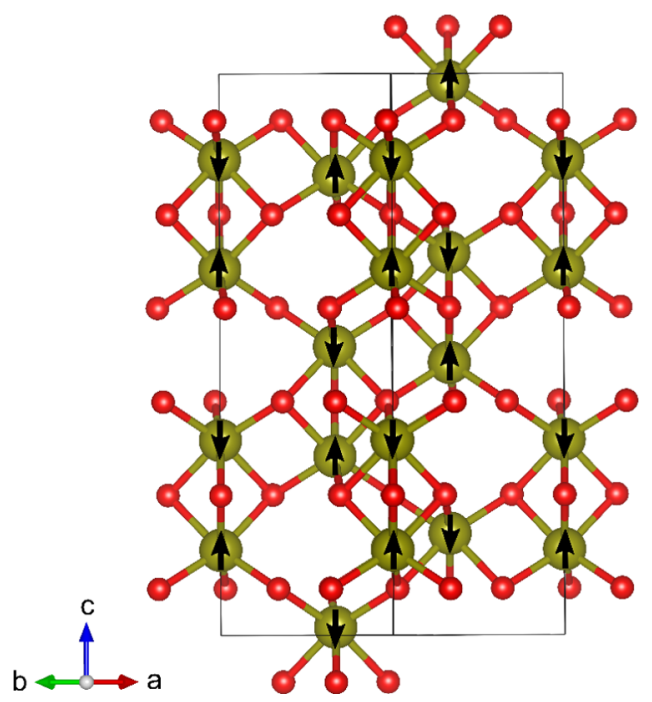

Figure 9. Lowest-energy unit cell for $\mathrm{V}_{2} \mathrm{O}_{3}$ predicted by USPEX (red: oxygen, yellow: vanadium). The directions of the magnetic moments are illustrated by arrows.

that corundum $\mathrm{V}_{2} \mathrm{O}_{3}$ structure transforms to a monoclinic structure at $\sim 150 \mathrm{~K}$, which, in turn, is known to be the most stable in an AFM configuration. ${ }^{75}$ However, monoclinic $\mathrm{V}_{2} \mathrm{O}_{3}$ structure has 20 atoms in the primitive unit cell and prediction of such structure with hybrid DFT methods would be computationally a very intensive effort (the local optimizations during USPEX structure search are run without any space group symmetry). On the basis of our PBE0/TZVP estimation at $0 \mathrm{~K}$, corundum $\mathrm{V}_{2} \mathrm{O}_{3}$ is less stable than the monoclinic structure only by $0.2 \mathrm{~kJ} / \mathrm{mol}$ per atom. Therefore, we carried out the USPEX search only for corundum-structured $\mathrm{V}_{2} \mathrm{O}_{3}$ in the AFM state $\left(\mathrm{V}_{4} \mathrm{O}_{6}\right.$ unit cell).

Overall, 122 and 214 candidate structures were screened in 10 and 16 generations, respectively (Figure 10a,b). The correct corundum $\mathrm{V}_{2} \mathrm{O}_{3}$ structure was found in the generation 1 in the first simulation and in the generation 7 in the second USPEX run.

The $\mathrm{V}_{2} \mathrm{O}_{3}$ example is a clear demonstration that USPEX can find the lowest-energy structure even in later generations without getting trapped in the local minima funnel. In fact, the $(+-+-)$ magnetic configuration was found to be the most stable for corundum $\mathrm{V}_{2} \mathrm{O}_{3}$ structure whereas such magnetic state is less favorable for previously described $\alpha$ - $\mathrm{Fe}_{2} \mathrm{O}_{3}$ (Figure 7). Comparison of the band gap is not feasible because corundum $\mathrm{V}_{2} \mathrm{O}_{3}$ is known to be a conducting material at the room temperature. Previously, the band gap for corundum $\mathrm{V}_{2} \mathrm{O}_{3}$ was calculated to be $2.7 \mathrm{eV}$ by local density approximation functional. ${ }^{77}$ Lattice constants of the calculated structure have differences of +2.1 and $1.2 \%$ for $a$ and $c$, respectively, compared with experiments. A similar difference was found for the $c / a$ ratio: 2.828 exp. and 2.736 calc. Experimental magnetic moments for corundum $\mathrm{V}_{2} \mathrm{O}_{3}$ have not been reported, and therefore it is not possible to compare with calculated values. However, we confirm that the predicted structure exactly reproduces corundum $\mathrm{V}_{2} \mathrm{O}_{3}$ that was calculated before. ${ }^{7}$
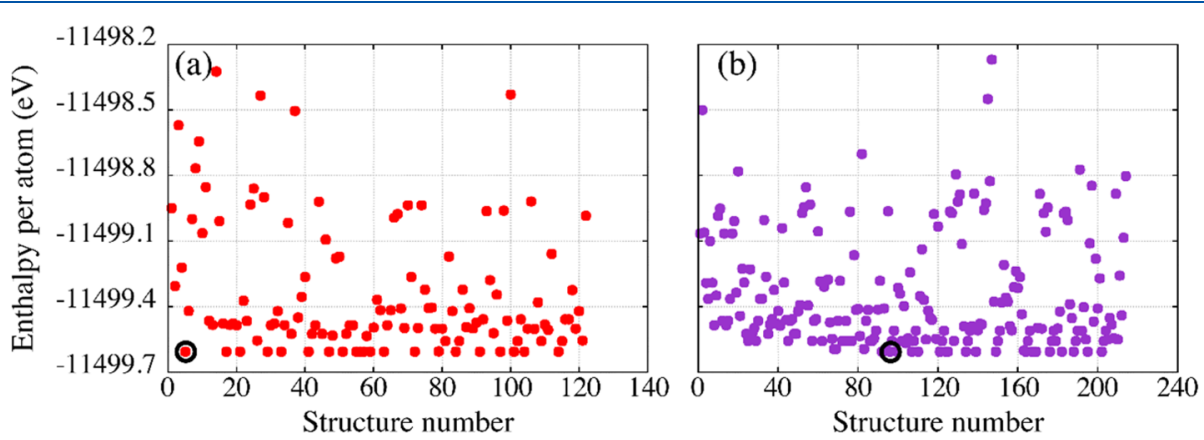

Figure 10. USPEX evolutionary crystal structure prediction of $\mathrm{V}_{2} \mathrm{O}_{3}$ (10 atoms in the unit cell), showing enthalpy per atom of all candidate structures along the evolutionary trajectory. Circle shows the first occurrence of the final global minimum. Plot (a) is the result of the first USPEX simulation and plot (b) is the results of the second USPEX simulation. 
(a)

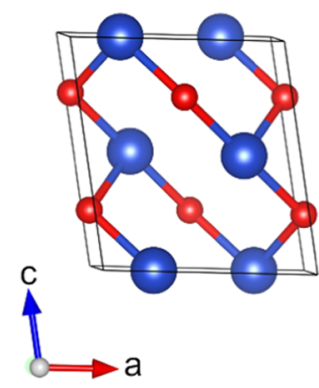

(b)

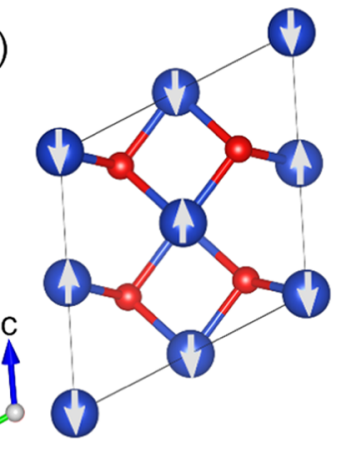

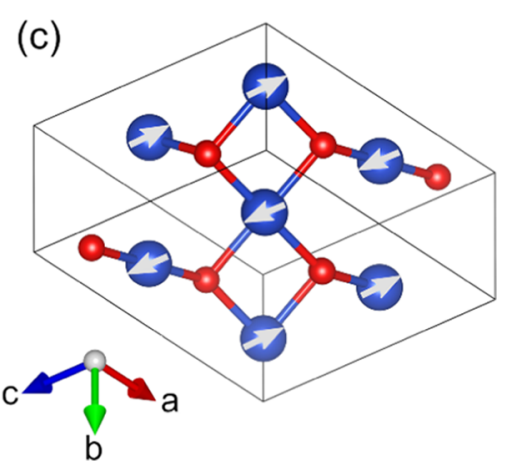

Figure 11. Lowest-energy unit cell of $\mathrm{CuO}$ predicted by USPEX (a) without and (b) with taking into account spins (red: oxygen, blue: copper). Structure $(\mathrm{c})$ is the $\mathrm{CuO}$ magnetic structure reported before and represents the lowest-energy spin configuration. The directions of the magnetic moments are illustrated by arrows.
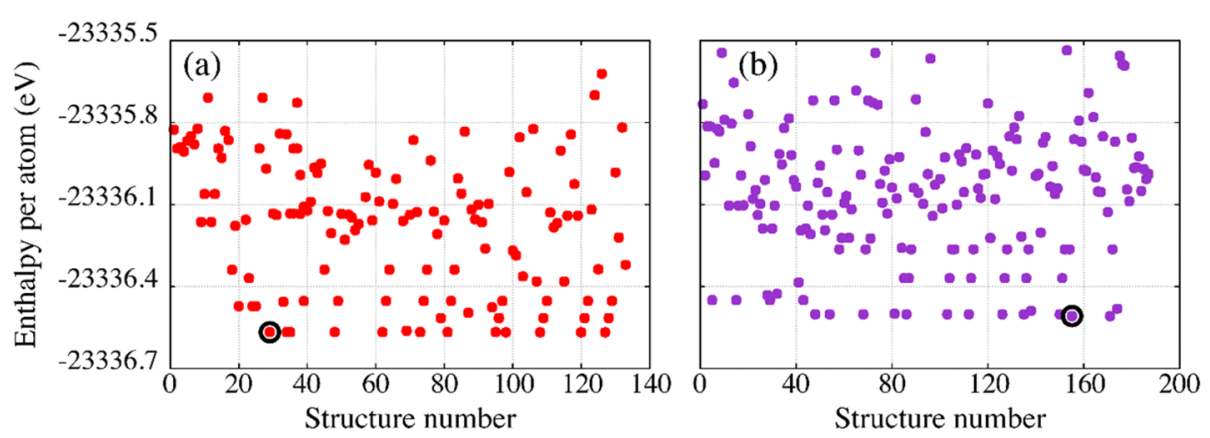

Figure 12. USPEX evolutionary crystal structure prediction of $\mathrm{CuO}$ (16 atoms in the unit cell), showing enthalpy per atom of all candidate structures along the evolutionary trajectory. The circle shows the first occurrence of the final global minimum. Plot (a) is the result of the first USPEX simulation, and plot (b) is the result of the second USPEX simulation using two relaxation steps.

3.6. CuO. Copper(II) oxide $(\mathrm{CuO})$ has a monoclinic structure with $C 2 / c$ space group (15) and four atoms in the primitive unit cell (Figure 11a). ${ }^{64,67}$ However, the primitive magnetic unit cell contains 16 atoms $\left(\mathrm{Cu}_{8} \mathrm{O}_{8}\right)$, which enables a huge number of spin configurations in the structure. ${ }^{78} \mathrm{CuO}$ is known to possess antiferromagnetic spin configuration below the Néel temperature of $230 \mathrm{~K}$. The magnetically ordered structure has a $P 2_{1} / c$ space group $(14)$ (Figure $\left.11 c\right) \cdot{ }^{65,66,78} \mathrm{Cu}$ atoms possess square-planar coordination, whereas the $\mathrm{O}$ atoms are almost tetrahedrally coordinated. The possibility for many different spin configurations together with a low space group makes the prediction of $\mathrm{CuO}$ magnetic structure the most challenging case studied here.

In general, 133 and 187 candidate structures within 10 and 13 generations were considered in the first (Figure 12a) and the second (Figure 12b) USPEX simulations, respectively.

The $\mathrm{CuO}$ structure reported here was identified in generations 3 and 12 for the first and the second USPEX runs, respectively. Evolutionary prediction of the magnetic structures with 16 atoms in the unit cell by using hybrid DFT is a time- and resource-consuming task. Therefore, we used only the first two relaxation steps instead of three for the second USPEX simulation, which can be observed on the basis of the energies from Figure 12: structures from the plot (b) have higher energies as expected. Notably, USPEX run with two relaxation steps screened much more candidate structures and both simulations resulted in the same lowest-energy structure. Band gap is overestimated by $1.7 \mathrm{eV}$. Predicted geometry is in good comparison with experimentally known structure with slightly overestimated lattice constants: $1.0,0.4$, and $0.4 \%$ for $a, b$, and $c$, respectively. ${ }^{67} \mathrm{Cu}-\mathrm{O}$ distance of the calculated structure is $1.97 \AA$, which is almost identical to 1.95 $\AA$ from the experiment.

The predicted $\mathrm{CuO}$ structure without taking into account spin configuration is identical to the experimentally known crystal structure (Figure 11a). The magnetic unit cell predicted by USPEX (Figure 11b) has some differences in spin-ordering resulting in a $P \overline{1}$ space group (2) compared with $\mathrm{CuO}$ structure reported before possessing a $P 2_{1} / c$ space group (14) ${ }^{78}$ but the energy difference between the predicted and reported before $\mathrm{CuO}$ structures is only $0.3 \mathrm{~kJ} / \mathrm{mol}$ per atom. This is a rather small value beyond the accuracy of the applied DFT-PBE0 method. Therefore, we consider that the predicted $\mathrm{CuO}$ structure is slightly different due to the limitations of DFT rather than the USPEX evolutionary algorithm itself. Notably, the magnetic moments of predicted $\mathrm{CuO}$ structure are in the range of the experimental values and the predicted magnetic structure has not been reported anywhere before.

\section{CONCLUSIONS}

We have carried out crystal structure predictions of magnetic transition binary metal oxides $\mathrm{NiO}, \mathrm{CoO}, \alpha-\mathrm{Fe}_{2} \mathrm{O}_{3}, \mathrm{~V}_{2} \mathrm{O}_{3}$, and $\mathrm{CuO}$ by using USPEX code and new CRYSTAL interface developed here. We reported the first successful USPEX predictions of magnetic structures by using hybrid DFT methods. Geometry, spin configurations, and magnetic moments of the studied structures are consistent with experimental data. The spin configuration predicted for $\mathrm{CuO}$ was found to be a little bit different compared to that of the most stable $\mathrm{CuO}$ structure, but the energy difference is so small that the prediction is limited by the accuracy of hybrid DFT, and it is not due to the evolutionary algorithm itself. To 
show that this approach can be used for nonmagnetic structures, we also carried out a successful prediction of the $\mathrm{Cu}_{2} \mathrm{O}$ crystal structure. We believe that the present benchmarks on magnetic transition binary metal oxides constitute a solid foundation toward further crystal structure prediction of novel magnetic materials.

\section{ASSOCIATED CONTENT}

\section{S Supporting Information}

The Supporting Information is available free of charge on the ACS Publications website at DOI: 10.1021/acs.jpcc.8b08238.

USPEX input files for predictions of $\mathrm{Cu}_{2} \mathrm{O}, \mathrm{NiO}, \mathrm{CoO}$, $\alpha-\mathrm{Fe}_{2} \mathrm{O}_{3}, \quad \mathrm{~V}_{2} \mathrm{O}_{3}$, and $\mathrm{CuO}$ structures; basis set information; CRYSTAL17 input files used in USPEX simulations; structural data of $\alpha-\mathrm{Fe}_{2} \mathrm{O}_{3}$ in the $(+--+)$ configuration and monoclinic $\mathrm{V}_{2} \mathrm{O}_{3}$ structures (PDF)

\section{AUTHOR INFORMATION}

\section{Corresponding Author}

*E-mail: mikhail.kuklin@aalto.fi.

\section{ORCID}

Mikhail S. Kuklin: 0000-0001-9289-6905

Antti J. Karttunen: 0000-0003-4187-5447

\section{Notes}

The authors declare no competing financial interest.

\section{ACKNOWLEDGMENTS}

Computing resources from CSC, the Finnish IT Center for Science, are gratefully acknowledged.

\section{REFERENCES}

(1) Schön, J. C.; Jansen, M. First Step Towards Planning of Syntheses in Solid-State Chemistry: Determination of Promising Structure Candidates by Global Optimization. Angew. Chem., Int. Ed. 1996, 35, 1286-1304.

(2) Schönborn, S. E.; Goedecker, S.; Roy, S.; Oganov, A. R. The Performance of Minima Hopping and Evolutionary Algorithms for Cluster Structure Prediction. J. Chem. Phys. 2009, 130, No. 144108.

(3) Martoňák, R.; Laio, A.; Parrinello, M. Predicting Crystal Structures: The Parrinello-Rahman Method Revisited. Phys. Rev. Lett. 2003, 90, No. 075503.

(4) Goedecker, S. Minima Hopping: An Efficient Search Method for the Global Minimum of the Potential Energy Surface of Complex Molecular Systems. J. Chem. Phys. 2004, 120, 9911-9917.

(5) Martonák, R.; Laio, A.; Bernasconi, M.; Ceriani, C.; Raiteri, P.; Zipoli, F.; Parrinello, M. Simulation of Structural Phase Transitions by Metadynamics. Z. Kristallogr. 2005, 220, 489-498.

(6) Day, G. M.; Motherwell, W. D. S.; Ammon, H. L.; Boerrigter, S. X. M.; Della Valle, R. G.; Venuti, E.; Dzyabchenko, A.; Dunitz, J. D.; Schweizer, B.; Van Eijck, B. P.; et al. A Third Blind Test of Crystal Structure Prediction. Acta Crystallogr. 2005, B61, 511-527.

(7) Maddox, J. Crystals from First Principles. Nature 1988, 335, 201.

(8) Freund, H. J.; Pacchioni, G. Oxide Ultra-Thin Films on Metals: New Materials for the Design of Supported Metal Catalysts. Chem. Soc. Rev. 2008, 37, 2224-2242.

(9) Nilius, N. Properties of Oxide Thin Films and Their Adsorption Behavior Studied by Scanning Tunneling Microscopy and Conductance Spectroscopy. Surf. Sci. Rep. 2009, 64, 595-659.

(10) Giordano, L.; Pacchioni, G. Oxide Films at the Nanoscale: New Structures, New Functions, and New Materials. Acc. Chem. Res. 2011, 44, 1244-1252.

(11) Honkala, K. Tailoring Oxide Properties: An Impact on Adsorption Characteristics of Molecules and Metals. Surf. Sci. Rep. 2014, 69, 366-388.
(12) Tripathi, T. S.; Karppinen, M. Atomic Layer Deposition of pType Semiconducting Thin Films: A Review. Adv. Mater. Interfaces 2017, 4, No. 1700300.

(13) Karttunen, A. J.; Tynell, T.; Karppinen, M. Layer-by-Layer Design of Nanostructured Thermoelectrics: First-Principles Study of $\mathrm{ZnO}$ : Organic Superlattices Fabricated by ALD/MLD. Nano Energy 2016, 22, 338-348.

(14) Linnera, J.; Sansone, G.; Maschio, L.; Karttunen, A. J. Thermoelectric Properties of p-Type $\mathrm{Cu}_{2} \mathrm{O}, \mathrm{CuO}$, and $\mathrm{NiO}$ from Hybrid Density Functional Theory. J. Phys. Chem. C 2018, 122, 15180-15189.

(15) Wang, L.; Maxisch, T.; Ceder, G. Oxidation Energies of Transition Metal Oxides within the GGA+U Framework. Phys. Rev. B 2006, 73, No. 115118.

(16) Schrön, A.; Rödl, C.; Bechstedt, F. Crystalline and Magnetic Anisotropy of the 3d-Transition Metal Monoxides $\mathrm{MnO}, \mathrm{FeO}, \mathrm{CoO}$, and NiO. Phys. Rev. B 2012, 86, No. 115134.

(17) Noh, J.; Osman, O. I.; Aziz, S. G.; Winget, P.; Brédas, J. L. A Density Functional Theory Investigation of the Electronic Structure and Spin Moments of Magnetite. Sci. Technol. Adv. Mater. 2014, 15, No. 044202.

(18) Lima, A. F. Density Functional Theory Study on the Magnetic Properties of $\mathrm{Co}_{3} \mathrm{O}_{4}$ with Normal Spinel Structure. J. Phys. Chem. Solids 2016, 91, 86-89.

(19) Singh, V.; Kosa, M.; Majhi, K.; Major, D. T. Putting DFT to the Test: A First-Principles Study of Electronic, Magnetic, and Optical Properties of $\mathrm{Co}_{3} \mathrm{O}_{4}$. J. Chem. Theory Comput. 2015, 11, 64-72.

(20) Deng, H. X.; Li, J.; Li, S. S.; Xia, J. B.; Walsh, A.; Wei, S. H. Origin of Antiferromagnetism in CoO: A Density Functional Theory Study. Appl. Phys. Lett. 2010, 96, No. 162508.

(21) Bredow, T.; Gerson, A. R. Effect of Exchange and Correlation on Bulk Properties of $\mathrm{MgO}, \mathrm{NiO}$, and CoO. Phys. Rev. B 2000, 61, 5194-5201.

(22) Rollmann, G.; Rohrbach, A.; Entel, P.; Hafner, J. FirstPrinciples Calculation of the Structure and Magnetic Phases of Hematite. Phys. Rev. B 2004, 69, No. 165107.

(23) Linnera, J.; Karttunen, A. J. Ab Initio Study of the Lattice Thermal Conductivity of $\mathrm{Cu}_{2} \mathrm{O}$ Using the Generalized Gradient Approximation and Hybrid Density Functional Methods. Phys. Rev. B 2017, 96, No. 014304

(24) Rödl, C.; Fuchs, F.; Furthmüller, J.; Bechstedt, F. Quasiparticle Band Structures of the Antiferromagnetic Transition-Metal Oxides $\mathrm{MnO}, \mathrm{FeO}, \mathrm{CoO}$, and NiO. Phys. Rev. B 2009, 79, No. 045102.

(25) Kulik, H. J.; Marzari, N. Transition-Metal Dioxides: A Case for the Intersite Term in Hubbard-Model Functionals. J. Chem. Phys. 2011, 134, No. 094103.

(26) Chen, X.; Parker, D.; Du, M. H.; Singh, D. J. Potential Thermoelectric Performance of Hole-Doped $\mathrm{Cu}_{2} \mathrm{O}$. New J. Phys. 2013, 15, No. 043029.

(27) Seo, D. H.; Urban, A.; Ceder, G. Calibrating Transition-Metal Energy Levels and Oxygen Bands in First-Principles Calculations: Accurate Prediction of Redox Potentials and Charge Transfer in Lithium Transition-Metal Oxides. Phys. Rev. B 2015, 92, No. 115118.

(28) Zhao, Q.; Kulik, H. J. Where Does the Density Localize in the Solid State? Divergent Behavior for Hybrids and DFT+U. J. Chem. Theory Comput. 2018, 14, 670-683.

(29) Gillen, R.; Robertson, J. Accurate Screened Exchange Band Structures for the Transition Metal Monoxides $\mathrm{MnO}, \mathrm{FeO}, \mathrm{CoO}$ and NiO. J. Phys.: Condens. Matter 2013, 25, No. 165502.

(30) Woodley, S. M.; Battle, P. D.; Gale, J. D.; Catlow, R. A. C. The Prediction of Inorganic Crystal Structures Using a Genetic Algorithm and Energy Minimisation. Phys. Chem. Chem. Phys. 1999, 1, 25352542.

(31) Woodley, S. M. Prediction of Crystal Structures Using Evolutionary Algorithms and Related Techniques. Struct. Bonding 2004, 110, 95-132.

(32) Oganov, A. R.; Glass, C. W. Crystal Structure Prediction Using $\mathrm{Ab}$ Initio Evolutionary Techniques: Principles and Applications. J. Chem. Phys. 2006, 124, No. 244704. 
(33) Oganov, A. R.; Lyakhov, A. O.; Valle, M. How Evolutionary Crystal Structure Prediction Works-and Why. Acc. Chem. Res. 2011, 44, 227-237.

(34) Lyakhov, A. O.; Oganov, A. R.; Stokes, H. T.; Zhu, Q. New Developments in Evolutionary Structure Prediction Algorithm USPEX. Comput. Phys. Commun. 2013, 184, 1172-1182.

(35) Glass, C. W.; Oganov, A. R.; Hansen, N. USPEX-Evolutionary Crystal Structure Prediction. Comput. Phys. Commun. 2006, 175, 713-720.

(36) Oganov, A. R.; Ma, Y.; Glass, C. W.; Valle, M. Evolutionary Crystal Structure Prediction: Overview of the USPEX Method and Some of Its Applications. Psi-k Newsl. 2007, 84, 142-171.

(37) Oganov, A. R.; Glass, C. W. Evolutionary Crystal Structure Prediction as a Tool in Materials Design. J. Phys.: Condens. Matter 2008, 20, No. 064210.

(38) Oganov, A. R.; Valle, M. How to Quantify Energy Landscapes of Solids. J. Chem. Phys. 2009, 130, No. 104504.

(39) Lyakhov, A. O.; Oganov, A. R.; Valle, M. How to Predict Very Large and Complex Crystal Structures. Comput. Phys. Commun. 2010, 181, 1623-1632.

(40) Oganov, A. R. Modern Methods of Crystal Structure Prediction; Wiley-VCH: Berlin, 2010.

(41) Dovesi, R.; Erba, A.; Orlando, R.; Zicovich-Wilson, C. M.; Civalleri, B.; Maschio, L.; Rérat, M.; Casassa, S.; Baima, J.; Salustro, S.; et al. Quantum-Mechanical Condensed Matter Simulations with CRYSTAL. Wiley Interdiscip. Rev.: Comput. Mol. Sci. 2018, 8, No. e1360.

(42) Perdew, J. P.; Burke, K.; Ernzerhof, M. Generalized Gradient Approximation Made Simple. Phys. Rev. Lett. 1996, 77, 3865-3868.

(43) Adamo, C.; Barone, V. Toward Reliable Density Functional Methods without Adjustable Parameters: The PBE0 Model. J. Chem. Phys. 1999, 110, 6158-6170.

(44) Weigend, F.; Ahlrichs, R. Balanced Basis Sets of Split Valence, Triple Zeta Valence and Quadruple Zeta Valence Quality for H to Rn: Design and Assessment of Accuracy. Phys. Chem. Chem. Phys. 2005, 7, 3297-3305.

(45) Dovesi, R.; Saunders, V. R.; Roetti, C.; Orlando, R.; ZicovichWilson, C. M.; Pascale, F.; Civalleri, B.; Doll, K.; Harrison, N. M.; Bush, I. J.; et al. CRYSTAL17 User's Manual; University of Torino: Torino, Italy, 2016.

(46) Kirfel, A.; Eichhorn, K. Accurate Structure Analysis with Synchrotron Radiation. The Electron Density in $\mathrm{Al}_{2} \mathrm{O}_{3}$ and $\mathrm{Cu}_{2} \mathrm{O}$. Acta Crystallogr. 1990, 46, 271-284.

(47) Meyer, B. K.; Polity, A.; Reppin, D.; Becker, M.; Hering, P.; Klar, P. J.; Sander, T.; Reindl, C.; Benz, J.; Eickhoff, M.; et al. Binary Copper Oxide Semiconductors: From Materials Towards Devices. Phys. Status Solidi B 2012, 249, 1487-1509.

(48) Alperin, H. A. The Magnetic Form Factor of Nickel Oxide. J. Phys. Soc. Jpn., Suppl. B. 1962, 17, 12-17.

(49) Fender, B. E. F.; Jacobson, A. J.; Wedgwood, F. A. Covalency Parameters in MnO, $\alpha$-MnS, and NiO. J. Chem. Phys. 1968, 48, 990994.

(50) Cheetham, A. K.; Hope, D. A. O. Magnetic Ordering and Exchange Effects in the Antiferromagnetic Solid Solutions $\mathrm{Mn}_{\mathrm{x}} \mathrm{Ni}_{1-\mathrm{x}} \mathrm{O}$. Phys. Rev. B 1983, 27, 6964-6967.

(51) Lide, D. CRC Handbook of Chemistry and Physics, 79th ed.; CRC Press: Boca Raton, 2005.

(52) Hüfner, S.; Osterwalder, J.; Riesterer, T.; Hulliger, F. Photoemission and Inverse Photoemission Spectroscopy of NiO. Solid State Commun. 1984, 52, 793-796.

(53) Sawatzky, G. A.; Allen, J. W. Magnitude and Origin of the Band Gap in NiO. Phys. Rev. Lett. 1984, 53, 2339-2342.

(54) Khan, D. C.; Erickson, R. A. Magnetic Form Factor of Co++ Ion in Cobaltous Oxide. Phys. Rev. B 1970, 1, 2243-2249.

(55) Herrmann-Ronzaud, D.; Burlet, P.; Rossat-Mignod, J. Equivalent Type-II Magnetic Structures: CoO, a Collinear Antiferromagnet. J. Phys. C: Solid State Phys. 1978, 11, 2123-2137.

(56) Sasaki, S.; Fujino, K.; Takéuchi, Y. X-Ray Determination of Electron-Density Distributions in Oxides, $\mathrm{MgO}, \mathrm{MnO}, \mathrm{CoO}$, and
$\mathrm{NiO}$, and Atomic Scattering Factors of Their Constituent Atoms. Proc. Jpn. Acad., Ser. B 1979, 55, 43-48.

(57) Parmigiani, F.; Sangaletti, L. Fine Structures in the X-Ray Photoemission Spectra of $\mathrm{MnO}, \mathrm{FeO}, \mathrm{CoO}$, and $\mathrm{NiO}$ Single Crystals. J. Electron Spectrosc. Relat. Phenom. 1999, 98-99, 287-302.

(58) Krén, E.; Szabó, P.; Konczos, G. Neutron Diffraction Studies on the $(1-x) \mathrm{Fe}_{2} \mathrm{O}_{3}-x \mathrm{Rh}_{2} \mathrm{O}_{3}$ System. Phys. Lett. 1965, 19, 103-104.

(59) Maslen, E. N.; Streltsov, V. A.; Streltsova, N. R.; Ishizawa, N. Synchrotron X-Ray Study of the Electron Density in $\alpha-\mathrm{Fe}_{2} \mathrm{O}_{3}$. Acta Crystallogr. 1994, 50, 435-441.

(60) Finger, L. W.; Hazen, R. M. Crystal Structure and Isothermal Compression of $\mathrm{Fe}_{2} \mathrm{O}_{3}, \mathrm{Cr}_{2} \mathrm{O}_{3}$, and $\mathrm{V}_{2} \mathrm{O}_{3}$ to 50 Kbars. J. Appl. Phys. 1980, 51, 5362-5367.

(61) Marusak, L. A.; Messier, R.; White, W. B. Optical Absorption Spectrum of Hematite, $\alpha-\mathrm{Fe}_{2} \mathrm{O}_{3}$ near IR to UV. J. Phys. Chem. Solids 1980, 41, 981-984.

(62) Strehlow, W. H.; Cook, E. L. Compilation of Energy Band Gaps in Elemental and Binary Compound Semiconductors and Insulators. J. Phys. Chem. Ref. Data 1973, 2, 163-200.

(63) Catti, M.; Sandrone, G.; Dovesi, R. Periodic Unrestricted Hartree-Fock Study of Corundumlike $\mathrm{Ti}_{2} \mathrm{O}_{3}$ and $\mathrm{V}_{2} \mathrm{O}_{3}$. Phys. Rev. B 1997, 55, 16122-16131.

(64) Forsyth, J. B.; Brown, P. J.; Wanklyn, B. M. Magnetism in Cupric Oxide. J. Phys. C: Solid State Phys. 1988, 21, 2917-2929.

(65) Yang, B. X.; Thurston, T. R.; Tranquada, J. M.; Shirane, G. Magnetic Neutron Scattering Study of Single-Crystal Cupric Oxide. Phys. Rev. B 1989, 39, 4343-4349.

(66) Yang, B. X.; Tranquada, J.; Shirane, G. Neutron Scattering Studies of the Magnetic Structure of Cupric Oxide. Phys. Rev. B 1988, $38,174-178$

(67) Åsbrink, S.; Norrby, L. J. A Refinement of the Crystal Structure of Copper(II) Oxide with a Discussion of Some Exceptional E.s.d.'s. Acta Crystallogr. 1970, B26, 8-15.

(68) Marabelli, F.; Parravicini, G. B.; Salghetti-Drioli, F. Optical Gap of CuO. Phys. Rev. B 1995, 52, 1433-1436.

(69) Anisimov, V. I.; Zaanen, J.; Andersen, O. K. Band Theory and Mott Insulators: Hubbard U Instead of Stoner I. Phys. Rev. B 1991, 44, 943-954.

(70) Kittel, C. Introduction to Solid State Physics, 6th ed.; John Wiley \& Sons: New York, 1986.

(71) de P. R. Moreira, I.; Illas, F.; Martin, R. L. Effect of Fock Exchange on the Electronic Structure and Magnetic Coupling in NiO. Phys. Rev. B 2002, 65, No. 155102.

(72) Skone, J. H.; Govoni, M.; Galli, G. Self-Consistent Hybrid Functional for Condensed Systems. Phys. Rev. B 2014, 89, No. 195112.

(73) Erba, A. Self-Consistent Hybrid Functionals for Solids: A FullyAutomated Implementation. J. Phys.: Condens. Matter 2017, 29, No. 314001.

(74) Sandratskii, L. M.; Uhl, M.; Kübler, J. Band Theory for Electronic and Magnetic Properties Of $\alpha-\mathrm{Fe}_{2} \mathrm{O}_{3}$. J. Phys.: Condens. Matter 1996, 8, 983-989.

(75) Dernier, P. D.; Marezio, M. Crystal Structure of the LowTemperature Antiferromagnetic Phase of $\mathrm{V}_{2} \mathrm{O}_{3}$. Phys. Rev. B 1970, 2, $3771-3776$

(76) Dernier, P. D. The Crystal Structure of $\mathrm{V}_{2} \mathrm{O}_{3}$ and $\left(\mathrm{V}_{0.962} \mathrm{Cr}_{0.0382}\right)_{2} \mathrm{O}_{3}$ near the Metal-Insulator Transition. J. Phys. Chem. Solids 1970, 31, 2569-2575.

(77) Mattheiss, L. F. Band Properties of Metallic Corundum-Phase $\mathrm{V}_{2} \mathrm{O}_{3}$. J. Phys.: Condens. Matter 1994, 6, 6477-6484.

(78) Rödl, C.; Sottile, F.; Reining, L. Quasiparticle Excitations in the Photoemission Spectrum of $\mathrm{CuO}$ from First Principles: A GW Study. Phys. Rev. B 2015, 91, No. 045102. 